\title{
RESEARCH
}

\section{Characterization of global fields by Dirichlet $L$-series}

\author{
Gunther Cornelissen ${ }^{1}$, Bart de Smit ${ }^{2}$, Xin Li ${ }^{3}$, Matilde Marcolli ${ }^{4,5,6}$ and Harry Smit ${ }^{{ }^{*} \text { (i) }}$
}

\author{
*Correspondence: \\ h.j.smit@uu.nl \\ ${ }^{1}$ Mathematisch Instituut, \\ Universiteit Utrecht, Postbus \\ 80.010, 3508 TA Utrecht, The \\ Netherlands \\ Full list of author information is \\ available at the end of the article \\ Part of this work was done whilst \\ the first and third author enjoyed \\ the hospitality of the University \\ of Warwick (special thanks to \\ Richard Sharp for making it \\ possible).
}

\begin{abstract}
We prove that two global fields are isomorphic if and only if there is an isomorphism of groups of Dirichlet characters that preserves $L$-series.
\end{abstract}

Keywords: Class field theory, L-series, Arithmetic equivalence

Mathematics Subject Classification: 11R37, 11R42, 11R56, 14H30

\section{Introduction}

As was discovered by Gaßmann in 1926 [10], number fields are not uniquely determined up to isomorphism by their zeta functions. A theorem of Tate [20] from 1966 implies the same for global function fields. At the other end of the spectrum, results of Neukirch and Uchida $[15,21,22]$ around 1970 state that the absolute Galois group does uniquely determine a global field. The better understood abelianized Galois group again does not determine the field up to isomorphism at all, as follows from the description of its character group by Kubota in 1957 ([13], compare [1,17]). Funakura ([9, $\mathbb{~ I ] , ~ u s i n g ~ [ 8 , ~ T h m . ~ 5 ] ) ~ h a s ~}$ shown in 1980 that there exists a number field $k$ (of degree 255! over $\mathbb{Q}$ ) and two nonisomorphic abelian extensions $\mathbb{K} / k$ and $\mathbb{L} / k$ of degree 4 with a bijection between all Artin $L$-series of $\mathbb{K} / k$ and $\mathbb{L} / k$ (or, equivalently, between the $L$-series of the four occurring characters).

In this paper, we prove that two global fields $\mathbb{K}$ and $\mathbb{L}$ are isomorphic if and only if there exists an isomorphism of groups of Dirichlet characters $\breve{\psi}: \breve{G}_{\mathbb{K}}^{\text {ab }} \stackrel{\sim}{\rightarrow} \breve{G}_{\mathbb{L}}^{\text {ab }}$ that preserves $L$-series: $L_{\mathbb{K}}(\chi)=L_{\mathbb{L}}(\breve{\psi}(\chi))$ for all $\chi \in \breve{G}_{\mathbb{K}}^{\text {ab }}$. A more detailed series of equivalences can be found in the Main Theorem 3.1 below. To connect this theorem to the discussion in the previous paragraph, observe that the existence of $\breve{\psi}$ without equality of $L$-series is the same as $\mathbb{K}$ and $\mathbb{L}$ having abelianized Galois groups that are isomorphic as topological groups, and that for the trivial character $\chi_{\text {triv }}$, we have $L_{\mathbb{K}}\left(\chi_{\text {triv }}\right)=\zeta_{\mathbb{K}}$, so that preserving $L$-series at $\chi_{\text {triv }}$ is the same as $\mathbb{K}$ and $\mathbb{L}$ having the same zeta function. Contrary to the result of Funakura, our characters do not factor over a fixed Galois group.

In global function fields we explicitly construct the isomorphism of function fields via a map of kernels of reciprocity maps (much akin to the final step in Uchida's proof [22]). For number fields, we do not need the full hypothesis: we can prove the stronger result that for every number field, there exists a character of any chosen order $>2$ for which the $L$-series does not equal any other Dirichlet $L$-series of any other field (see Theorem 10.1).

(c) The Author(s) 2018. This article is distributed under the terms of the Creative Commons Attribution 4.0 International License (http://creativecommons.org/licenses/by/4.0/), which permits unrestricted use, distribution, and reproduction in any medium, provided you give appropriate credit to the original author(s) and the source, provide a link to the Creative Commons license, and indicate if changes were made. 
The method here is not via the kernel of the reciprocity map, but rather via representation theory.

We briefly indicate the relation of our work to previous results. The main result was first stated in a 2010 preprint by two of the current authors, who discovered it through methods from mathematical physics ([4], now split into two parts [5] and [6]). For function fields, the main result was proven by one of the authors in [3], using dynamical systems, referring, however, to [4] for some auxiliary results, of which we present the first published proofs in the current paper. Sections 9 and 10 contain an independent proof for the number field case that was found by the second author in 2011 . The current paper not only presents full and simplified proofs, but also uses only classical methods from number theory (class field theory, Chebotarev, Grunwald-Wang, and inverse Galois theory). In [19], reconstruction of field extensions of a fixed rational function field from relative $L$-series is treated from the point of view of the method in Sects. 9 and 10 of this paper.

After some preliminaries in the first section, we state the main result in Sect. 3. The next few sections outline the proof of the various equivalences in the main theorem, using basic class field theory and work of Uchida and Hoshi. In the final sections, we deal with number fields, and use representation theory to prove some stronger results.

\section{Preliminaries}

In this section, we set notation and introduce the main object of study.

A monoid is a semigroup with identity element. If $R$ is a ring, we let $R^{*}$ denote its group of invertible elements.

Given a global field $\mathbb{K}$, we use the word prime to denote a non-zero prime ideal if $\mathbb{K}$ is a number field, and to denote an irreducible effective divisor if $\mathbb{K}$ is a global function field. Let $\mathscr{P}_{\mathbb{K}}$ be the set of primes of $\mathbb{K}$. If $\mathfrak{p} \in \mathscr{P}_{\mathbb{K}}$, let $v_{\mathfrak{p}}$ be the normalized (additive) valuation corresponding to $\mathfrak{p}, \mathbb{K}_{\mathfrak{p}}$ the local field at $\mathfrak{p}$, and $\mathscr{O}_{\mathfrak{p}}$ its ring of integers. Let $\mathbb{A}_{\mathbb{K}, f}$ be the finite adele ring of $\mathbb{K}, \widehat{\mathscr{O}}_{\mathbb{K}}$ its ring of finite integral adeles and $\mathbb{A}_{\mathbb{K}, f}^{*}$ the group of ideles (invertible finite adeles), all with their usual topology. Note that in the function field case, infinite places do not exist, so that in that case, $\mathbb{A}_{\mathbb{K}, f}=\mathbb{A}_{\mathbb{K}}$ is the adele ring, $\widehat{\mathscr{O}}_{\mathbb{K}}$ is the ring of integral adeles and $\mathbb{A}_{\mathbb{K}, f}^{*}=\mathbb{A}_{\mathbb{K}}^{*}$ is the full group of ideles. If $\mathbb{K}$ is a number field, we denote by $\mathscr{O}_{\mathbb{K}}$ its ring of integers. If $\mathbb{K}$ is a global function field, we denote by $q$ the cardinality of the constant field.

Let $I_{\mathbb{K}}$ be the multiplicative monoid of non-zero integral ideals/effective divisors of our global field $\mathbb{K}$, so $I_{\mathbb{K}}$ is generated by $\mathscr{P}_{\mathbb{K}}$. We extend the valuation to ideals: if $\mathfrak{m} \in I_{\mathbb{K}}$ and $\mathfrak{p} \in \mathscr{P}_{\mathbb{K}}$, we define $v_{\mathfrak{p}}(\mathfrak{m}) \in \mathbb{Z}_{\geq 0}$ by requiring $\mathfrak{m}=\prod_{\mathfrak{p} \in \mathscr{P}_{\mathbb{K}}} \mathfrak{p}^{v_{\mathfrak{p}}(\mathfrak{m})}$. Let $N$ be the norm function on the monoid $I_{\mathbb{K}}$ : it is the multiplicative function defined on primes $\mathfrak{p}$ by $N(\mathfrak{p}):=\# \mathscr{O}_{\mathbb{K}} / \mathfrak{p}$ if $\mathbb{K}$ is a number field and $N(\mathfrak{p})=q^{\operatorname{deg}(\mathfrak{p})}$ if $\mathbb{K}$ is a function field. Given two global fields $\mathbb{K}$ and $\mathbb{L}$, we call a monoid homomorphism $\varphi: I_{\mathbb{K}} \rightarrow I_{\mathbb{L}}$ norm-preserving if $N(\varphi(\mathfrak{m}))=N(\mathfrak{m})$ for all $\mathfrak{m} \in I_{\mathbb{K}}$.

We have a surjective monoid homomorphism

$$
(\cdot)_{\mathbb{K}}: \mathbb{A}_{\mathbb{K}, f}^{*} \cap \widehat{\mathscr{O}}_{\mathbb{K}} \rightarrow I_{\mathbb{K}},\left(x_{\mathfrak{p}}\right)_{\mathfrak{p}} \mapsto \prod_{\mathfrak{p}} \mathfrak{p}^{\nu_{\mathfrak{p}}\left(x_{\mathfrak{p}}\right)}
$$

a restriction of the usual homomorphism from ideles to fractional ideals-we use the monoid version in Sect. 6.1 and in the companion paper [6]. A splitfor $(\cdot)_{\mathbb{K}}$ is by definition a monoid homomorphism $s_{\mathbb{K}}: I_{\mathbb{K}} \rightarrow \mathbb{A}_{\mathbb{K}, f}^{*} \cap \widehat{\mathscr{O}}_{\mathbb{K}}$ such that for every prime $\mathfrak{p}, s_{\mathbb{K}}(\mathfrak{p})=$ $\left(\ldots, 1, \pi_{\mathfrak{p}}, 1, \ldots\right)$ for some uniformizer $\pi_{\mathfrak{p}} \in \mathbb{K}_{\mathfrak{p}}$. It follows that $(\cdot)_{\mathbb{K}} \circ s_{\mathbb{K}}=\mathrm{id}_{I_{\mathbb{K}}}$. 
Let $G_{\mathbb{K}}^{\mathrm{ab}}$ be the Galois group of a maximal abelian extension $\mathbb{K}^{\mathrm{ab}}$ of $\mathbb{K}$, a profinite topological group. There is an Artin reciprocity map $\mathbb{A}_{\mathbb{K}}^{*} \rightarrow G_{\mathbb{K}}^{\text {ab }}$. In the number field case, we embed $\mathbb{A}_{\mathbb{K}, f}^{*}$ into the group of ideles $\mathbb{A}_{\mathbb{K}}^{*}$ via $\mathbb{A}_{\mathbb{K}, f}^{*} \ni x \mapsto(1, x) \in \mathbb{A}_{\mathbb{K}}^{*}$, restrict the Artin reciprocity map to $\mathbb{A}_{\mathbb{K}, f}^{*}$ and call this restriction $\operatorname{rec}_{\mathbb{K}}$. In the function field case, rec $\mathbb{K}$ is just the (full) Artin reciprocity map.

Let $\mathbb{T}$ denote the unit circle, equipped with the usual topology, and $\check{G}_{\mathbb{K}}^{\mathrm{ab}}=\operatorname{Hom}\left(G_{\mathbb{K}}^{\mathrm{ab}}, \mathbb{T}\right)$ the group of continuous linear characters of $G_{\mathbb{K}}^{\mathrm{ab}}$. Given $\chi \in \breve{G}_{\mathbb{K}}^{\mathrm{ab}}$, we write

$$
U(\chi):=\left\{\mathfrak{p} \in \mathscr{P}_{\mathbb{K}}:\left.\chi\right|_{\operatorname{rec}_{\mathbb{K}}\left(\mathscr{O}_{\mathfrak{p}}^{*}\right)}=1\right\}
$$

for the set of primes where $\chi$ is unramified. We denote by $\langle U(\chi)\rangle$ the submonoid of $I_{\mathbb{K}}$ generated by $U(\chi)$, i.e., $\mathfrak{m} \in I_{\mathbb{K}}$ is in $\langle U(\chi)\rangle$ if and only if $v_{\mathfrak{p}}(\mathfrak{m})=0$ for all $\mathfrak{p} \in \mathscr{P}_{\mathbb{K}} \backslash U(\chi)$. For $\mathfrak{m} \in\langle U(\chi)\rangle$, we set (in a well-defined way, as the choice of $s_{\mathbb{K}}$ is up to an element of $\widehat{\mathscr{O}}_{\mathbb{K}}^{*}$ ):

$$
\chi(\mathfrak{m}):=\chi\left(\operatorname{rec}_{\mathbb{K}}\left(s_{\mathbb{K}}(\mathfrak{m})\right)\right),
$$

and for $\mathfrak{m} \in I_{\mathbb{K}} \backslash\langle U(\chi)\rangle$ we set $\chi(\mathfrak{m})=0$.

For any $\chi \in \breve{G}_{\mathbb{K}}^{\text {ab }}$, the kernel ker $\chi$ is an open subgroup of $G_{\mathbb{K}}^{\text {ab }}$. Therefore, the fixed field of $\chi$, denoted $\mathbb{K}_{\chi}$, is a finite abelian extension of $\mathbb{K}$. Even more: as the extension is finite, there is an $n$ such that $\chi^{n}$ is the trivial character. It follows that $\operatorname{im} \chi$ is a subgroup of the $n^{\text {th }}$ roots of unity, hence cyclic. As we have an isomorphism im $\chi \stackrel{\sim}{\rightarrow} \operatorname{Gal}\left(\mathbb{K}_{\chi} / \mathbb{K}\right)$, we obtain that $\mathbb{K}_{\chi} / \mathbb{K}$ is a finite cyclic extension. Conversely, for any finite cyclic extension $\mathbb{K}^{\prime}$ of $\mathbb{K}$ there exists a character $\chi \in \breve{G}_{\mathbb{K}}^{\mathrm{ab}}$ such that $\operatorname{ker} \chi=\mathrm{Gal}\left(\mathbb{K}^{\mathrm{ab}} / \mathbb{K}^{\prime}\right)$.

The following two lemmas are easy, but we include a proof in the terminology of this paper for lack of a suitable reference.

Lemma 2.1 Let $\chi \in \breve{G}_{\mathbb{K}}^{\mathrm{ab}}$. The primes in $U(\chi)$ are exactly the primes that are unramified in $\mathbb{K}_{\chi}$.

Proof The character $\chi$ factors through the quotient map $G_{\mathbb{K}}^{\mathrm{ab}} \rightarrow \mathrm{Gal}\left(\mathbb{K}_{\chi} / \mathbb{K}\right)$, giving an injective character $\bar{\chi}: \operatorname{Gal}\left(\mathbb{K}_{\chi} / \mathbb{K}\right) \rightarrow \mathbb{T}$. Under the quotient map, the group $\operatorname{rec}_{\mathbb{K}}\left(\mathscr{O}_{\mathfrak{p}}^{*}\right)$ is mapped surjectively to the inertia group $I_{\mathfrak{p}}\left(\mathbb{K}_{\chi} / \mathbb{K}\right)$, hence we have $\chi\left(\operatorname{rec}_{\mathbb{K}}\left(\mathscr{O}_{\mathfrak{p}}^{*}\right)\right)=$ $\bar{\chi}\left(I_{\mathfrak{p}}\left(\mathbb{K}_{\chi} / \mathbb{K}\right)\right)$. As $\bar{\chi}$ is injective, this group is equal to $\{1\}$ precisely when the inertia group is trivial, i.e. when $\mathfrak{p}$ is unramified in $\mathbb{K}_{\chi}$.

Lemma 2.2 For any prime $\mathfrak{p} \in \mathscr{P}_{\mathbb{K}}$, set $N_{\mathfrak{p}}:=\bigcap_{\chi: \mathfrak{p} \in U(\chi)} \operatorname{ker} \chi$. Then $\operatorname{rec}_{\mathbb{K}}\left(\mathscr{O}_{\mathfrak{p}}^{*}\right)=N_{\mathfrak{p}}$, and the associated fixed field is equal to $\mathbb{K}^{\mathrm{ur}, \mathfrak{p}}$, the maximal abelian extension of $\mathbb{K}$ unramified at $\mathrm{p}$.

Proof By definition of $U(\chi)$, for any $\chi \in \breve{G}_{\mathbb{K}}^{\text {ab }}$ with $\mathfrak{p} \in U(\chi)$ we have $\operatorname{rec}_{\mathbb{K}}\left(\mathscr{O}_{\mathfrak{p}}^{*}\right) \subseteq \operatorname{ker} \chi$, hence $\operatorname{rec}_{\mathbb{K}}\left(\mathscr{O}_{\mathfrak{p}}^{*}\right) \subseteq N_{\mathfrak{p}}$. As $\mathscr{O}_{\mathfrak{p}}^{*}$ is compact and $\operatorname{rec}_{\mathbb{K}}$ is continuous, $\operatorname{rec}_{\mathbb{K}}\left(\mathscr{O}_{\mathfrak{p}}^{*}\right)$ is compact, and as $G_{\mathbb{K}}^{\text {ab }}$ is Hausdorff, $\operatorname{rec}_{\mathbb{K}}\left(\mathscr{O}_{\mathfrak{p}}^{*}\right)$ is closed.

Let $\mathbb{K}_{\mathscr{O}_{\mathfrak{p}}^{*}}$ denote the fixed field of $\operatorname{rec}_{\mathbb{K}}\left(\mathscr{O}_{\mathfrak{p}}^{*}\right)$. Under the quotient map $G_{\mathbb{K}}^{\mathrm{ab}} \rightarrow$ $\operatorname{Gal}\left(\mathbb{K}_{\mathscr{O}_{\mathfrak{p}}^{*}} / \mathbb{K}\right), \operatorname{rec}_{\mathbb{K}}\left(\mathscr{O}_{\mathfrak{p}}^{*}\right)$ is mapped to the inertia group $I_{\mathfrak{p}}\left(\mathbb{K}_{\mathscr{O}_{\mathfrak{p}}^{*}} / \mathbb{K}\right)$ by class field theory, but it is also mapped to $\{1\}$ by definition. Hence the inertia group is trivial, and

$$
\left(\mathbb{K}^{\mathrm{ab}}\right)^{\mathrm{rec}_{\mathbb{K}}\left(\mathscr{O}_{\mathfrak{p}}^{*}\right)}=\mathbb{K}_{\mathscr{O}_{\mathfrak{p}}^{*}} \subseteq \mathbb{K}^{\mathrm{ur}, \mathfrak{p}} .
$$

The extension associated to $N_{\mathfrak{p}}$ contains the composite of the extensions $\mathbb{K}_{\chi}$ associated to the individual $\chi$. The field $\mathbb{K}^{\text {ur, } \mathfrak{p}}$ is a composite of finite cyclic extensions of $\mathbb{K}$ unramified 
at $\mathfrak{p}$. As mentioned, for any such a finite cyclic extension $\mathbb{K}^{\prime}$ there exists a $\chi$ such that $\operatorname{ker} \chi=\operatorname{Gal}\left(\mathbb{K}^{\mathrm{ab}} / \mathbb{K}^{\prime}\right)$. Because this extension is unramified at $\mathfrak{p}$, the previous lemma asserts that $\mathfrak{p} \in U(\chi)$. It follows that

$$
\mathbb{K}^{\mathrm{ur}, \mathfrak{p}} \subseteq\left(\mathbb{K}^{\mathrm{ab}}\right)^{N_{\mathfrak{p}}} .
$$

Combining these two results, we obtain $\operatorname{rec}_{\mathbb{K}}\left(\mathscr{O}_{\mathfrak{p}}^{*}\right) \supseteq N_{\mathfrak{p}}$, and the result follows.

The (Dirichlet) L-function attached to a character $\chi$ of $\mathbb{K}$ is the function of the complex variable $s$ :

$$
L_{\mathbb{K}}(\chi)=\prod_{\mathfrak{p} \in U(\chi)}\left(1-\chi(\mathfrak{p}) N(\mathfrak{p})^{-s}\right)^{-1}=\sum_{\mathfrak{m} \in\langle U(\chi)\rangle} \chi(\mathfrak{m}) N(\mathfrak{m})^{-s} .
$$

(We drop the field $\mathbb{K}$ from the notation for the $L$-series unless confusion might arise.)

Let $\chi$ be a character with associated extension $\mathbb{K}_{\chi}$. For the primes in $U(\chi)$ we have that $\operatorname{rec}_{\mathbb{K}}\left(s_{\mathbb{K}}(\mathfrak{p})\right) \bmod$ ker $\chi$ is independent of the choice of the split $s_{\mathbb{K}}$, and equal to the Frobenius $\operatorname{Frob}_{\mathfrak{p}}$ in $\mathrm{Gal}\left(\mathbb{K}^{\prime} / \mathbb{K}\right)$; note that in a general extension, "the" Frobenius of an unramified prime in $\mathbb{K}$ is a conjugacy class in the Galois group, but in our setting of abelian extensions, it is an actual element. The $L$-series can thus be written as

$$
L(\chi)=\prod_{\mathfrak{p} \in U(\chi)}\left(1-\chi\left(\operatorname{Frob}_{\mathfrak{p}}\right) N(\mathfrak{p})^{-s}\right)^{-1} .
$$

\section{The main theorem}

Theorem 3.1 Let $\mathbb{K}$ and $\mathbb{L}$ be two global fields. The following are equivalent:

(i) There exists

- a monoid isomorphism $\varphi: I_{\mathbb{K}} \stackrel{\sim}{\rightarrow} I_{\mathbb{L}}$,

- an isomorphism of topological groups $\psi: G_{\mathbb{K}}^{\mathrm{ab}} \stackrel{\sim}{\rightarrow} G_{\mathbb{L}}^{\mathrm{ab}}$, and

- splits $s_{\mathbb{K}}: I_{\mathbb{K}} \rightarrow \mathbb{A}_{\mathbb{K}, f}^{*} \cap \widehat{\mathscr{O}}_{\mathbb{K}}, s_{\mathbb{L}}: I_{\mathbb{L}} \rightarrow \mathbb{A}_{\mathbb{L}, f}^{*} \cap \widehat{\mathscr{O}}_{\mathbb{L}}$

such that

$$
\begin{aligned}
& \psi\left(\operatorname{rec}_{\mathbb{K}}\left(\mathscr{O}_{\mathfrak{p}}^{*}\right)\right)=\operatorname{rec}_{\mathbb{L}}\left(\mathscr{O}_{\varphi(\mathfrak{p})}^{*}\right) \text { for every prime } \mathfrak{p} \text { of } \mathbb{K} \\
& \psi\left(\operatorname{rec}_{\mathbb{K}}\left(s_{\mathbb{K}}(\mathfrak{m})\right)\right)=\operatorname{rec}_{\mathbb{L}}\left(s_{\mathbb{L}}(\varphi(\mathfrak{m}))\right) \text { for all } \mathfrak{m} \in I_{\mathbb{K}}
\end{aligned}
$$

(ii) There exists

- a norm-preserving monoid isomorphism $\varphi: I_{\mathbb{K}} \stackrel{\sim}{\rightarrow} I_{\mathbb{L}}$, and

- an isomorphism of topological groups $\psi: G_{\mathbb{K}}^{\mathrm{ab}} \stackrel{\sim}{\longrightarrow} G_{\mathbb{L}}^{\mathrm{ab}}$

such that for every finite abelian extension $\mathbb{K}^{\prime}=\left(\mathbb{K}^{\mathrm{ab}}\right)^{N}$ of $\mathbb{K}$ ( $N$ a subgroup in $G_{\mathbb{K}}^{\mathrm{ab}}$ ) with corresponding field extension $\mathbb{L}^{\prime}=\left(\mathbb{L}^{\mathrm{ab}}\right)^{\psi(N)}$ of $\mathbb{L}, \varphi$ is a bijection between the unramified primes of $\mathbb{K}^{\prime} / \mathbb{K}$ and $\mathbb{L}^{\prime} / \mathbb{L}$ such that

$$
\psi\left(\operatorname{Frob}_{\mathfrak{p}}\right)=\operatorname{Frob}_{\varphi(\mathfrak{p})} \text { in } \operatorname{Gal}\left(\mathbb{L}^{\prime} / \mathbb{L}\right) .
$$

(iii) There exists an isomorphism of topological groups $\psi: G_{\mathbb{K}}^{\mathrm{ab}} \stackrel{\sim}{\rightarrow} G_{\mathbb{L}}^{\mathrm{ab}}$ such that

$$
L(\chi)=L(\breve{\psi}(\chi)) \text { for all } \chi \in \check{G}_{\mathbb{K}}^{\mathrm{ab}},
$$

where $\breve{\psi}$ is given by $\breve{\psi}(\chi)=\chi \circ \psi^{-1}$.

(iv) $\mathbb{K}$ and $\mathbb{L}$ are isomorphic as fields. 
We will refer to condition (i) as a reciprocity isomorphism, condition (ii) as a finite reciprocity isomorphism, and condition (iii) as an L-isomorphism. We will prove the implication (i) $\Rightarrow$ (ii) in Proposition 4.2, implication (ii) $\Rightarrow$ (iii) in Proposition 5.1, and implication (iii) $\Rightarrow$ (i) in Proposition 6.1. The equivalence with (iv) is proven in 8.1 for function fields and follows from 10.1 for number fields.

\section{From reciprocity isomorphism to finite reciprocity isomorphism}

Proposition 4.1 Assume 3.1(i). Then $\varphi$ is norm-preserving, i.e.

$$
N(\mathfrak{p})=N(\varphi(\mathfrak{p})) \text { for all } \mathfrak{p} \in \mathscr{P}_{\mathbb{K}}
$$

Proof We have

$$
\mathscr{O}_{\mathfrak{p}}^{*} \stackrel{\sim}{\longrightarrow} \operatorname{rec}_{\mathbb{K}}\left(\mathscr{O}_{\mathfrak{p}}^{*}\right) \stackrel{\sim}{\longrightarrow} \operatorname{rec}_{\mathbb{L}}\left(\mathscr{O}_{\varphi(\mathfrak{p})}^{*}\right) \stackrel{\sim}{\longrightarrow} \mathscr{O}_{\varphi(\mathfrak{p})}^{*}
$$

for all $\mathfrak{p} \in \mathscr{P}_{\mathbb{K}}$ as the local reciprocity map is injective. Thus, it suffices to find a way to read off $N(\mathfrak{p})$ from the isomorphism type of $\mathscr{O}_{\mathfrak{p}}^{*}$. Let tors $(A)$ be the torsion subgroup of an abelian group $A$, and let $A_{p}$ be the $p$-primary part of a finite abelian group $A$. Assume that $N(\mathfrak{p})=p^{f}$ for some prime $p$ and $f \geq 1$. The following facts follow from [16, Chapter II, Proposition (5.7)]:

- $p$ is uniquely determined by the property $\left(\mathscr{O}_{\mathfrak{p}}^{*} / \operatorname{tors}\left(\mathscr{O}_{\mathfrak{p}}^{*}\right)\right)^{p} \neq \mathscr{O}_{\mathfrak{p}}^{*} / \operatorname{tors}\left(\mathscr{O}_{\mathfrak{p}}^{*}\right)$,

- $N(\mathfrak{p})=p^{f}=\left|\operatorname{tors}\left(\mathscr{O}_{\mathfrak{p}}^{*}\right) / \operatorname{tors}\left(\mathscr{O}_{\mathfrak{p}}^{*}\right)_{p}\right|+1$.

So we indeed have (4).

Proposition 4.2 Condition 3.1(i) implies condition 3.1(ii).

Proof A prime $\mathfrak{p}$ of $\mathbb{K}$ is unramified in $\mathbb{K}^{\prime}$ if and only if $\operatorname{rec}_{\mathbb{K}}\left(\mathscr{O}_{\mathfrak{p}}^{*}\right) \subseteq N$. Therefore, (1) implies that $\varphi(\mathfrak{p})$ is unramified in $\mathbb{L}^{\prime}$, and the bijection follows by symmetry. Also, $\operatorname{rec}_{\mathbb{K}}\left(s_{\mathbb{K}}(\mathfrak{p})\right) \bmod N$ is independent of the choice of the split $s_{\mathbb{K}}$, and equal to the Frobenius $\operatorname{Frob}_{\mathfrak{p}}$ in $\operatorname{Gal}\left(\mathbb{K}^{\prime} / \mathbb{K}\right)$. Hence from (2) we obtain $\psi\left(\operatorname{Frob}_{\mathfrak{p}}\right)=\operatorname{Frob}_{\varphi(\mathfrak{p})}$. This proves (ii).

\section{From finite reciprocity isomorphism to L-isomorphism}

Proposition 5.1 Assume 3.1(ii). Then $L(\chi)=L(\breve{\psi}(\chi))$ for all $\chi \in \breve{G}_{\mathbb{K}}^{\text {ab }}$.

Proof Let $\chi \in \breve{G}_{\mathbb{K}}^{\mathrm{ab}}$, and let $\mathbb{K}_{\chi}=\left(\mathbb{K}^{\mathrm{ab}}\right)^{\mathrm{ker} \chi}$ denote the fixed field of ker $\chi$. As mentioned in Sect. 2, the associated $L$-series can be written as

$$
L(\chi)=\prod_{\mathfrak{p} \in U(\chi)}\left(1-\chi\left(\operatorname{Frob}_{\mathfrak{p}}\right) N(\mathfrak{p})^{-s}\right)^{-1} .
$$

We have $\psi(\operatorname{ker} \chi)=\operatorname{ker}(\breve{\psi}(\chi))$ by definition of $\breve{\psi}$. Let $\mathbb{L}_{\breve{\psi}(\chi)}=\left(\mathbb{L}^{\mathrm{ab}}\right)^{\psi(\operatorname{ker} \chi)}$. Let $\mathfrak{p} \in \mathscr{P}_{\mathbb{K}}$ and $\mathfrak{q}:=\varphi(\mathfrak{p}) \in \mathscr{P}_{\mathbb{L}}$. By assumption, $\varphi(U(\chi))=U(\breve{\psi}(\chi)), N(\mathfrak{p})=N(\mathfrak{q})$ and if $\mathfrak{p} \in U(\chi)$ then $\psi\left(\right.$ Frob $\left._{\mathfrak{p}}\right)=$ Frob $_{\mathfrak{q}}$ and thus $\chi\left(\operatorname{Frob}_{\mathfrak{p}}\right)=\breve{\psi}(\chi)\left(\right.$ Frob $\left._{\mathfrak{q}}\right)$. It follows that 


$$
\begin{aligned}
L(\chi) & =\prod_{\mathfrak{p} \in U(\chi)}\left(1-\chi\left(\operatorname{Frob}_{\mathfrak{p}}\right) N(\mathfrak{p})^{-s}\right)^{-1} \\
& =\prod_{\mathfrak{q} \in U(\breve{\psi}(\chi))}\left(1-\breve{\psi}(\chi)\left(\operatorname{Frob}_{\mathfrak{q}}\right) N(\mathfrak{q})^{-s}\right)^{-1} \\
& =L(\breve{\psi}(\chi)) .
\end{aligned}
$$

\section{From L-isomorphism to reciprocity isomorphism}

Proposition 6.1 Assume that there exists an isomorphism of topological groups $\psi$ : $G_{\mathbb{K}}^{\mathrm{ab}} \stackrel{\sim}{\rightarrow} G_{\mathbb{L}}^{\mathrm{ab}}$ with $L(\chi)=L(\breve{\psi}(\chi))$ for all $\chi \in \breve{G}_{\mathbb{K}}^{\mathrm{ab}}$. Then 3.1(i) holds.

Proof The idea of the proof is to construct a bijection of primes $\varphi$ with the desired properties via the use of a type of characters that have value 1 on all but one of the primes of a certain norm.

For the proof, which will occupy this entire section, we will use the following notation. Whenever an object has subscript $N,<N$, or $\geq N$, all associated sets of (prime) ideals are restricted to (prime) ideals of norm $N,<N$ or $\geq N$ respectively. For example, $U_{N}(\chi)$ is the set of primes of norm $N$ at which $\chi$ is unramified. We use the multiplicative form of the $L$-series, i.e.

$$
L_{<N}(\chi)=\prod_{\mathfrak{p} \in U_{<N}(\chi)}\left(1-\chi\left(\operatorname{Frob}_{\mathfrak{p}}\right) N(\mathfrak{p})^{-s}\right)^{-1}
$$

and $L_{\geq N}(\chi)$ is defined similarly.

Our approach is as follows: for every $N \in \mathbb{N}$ we construct a bijection

$$
\varphi_{N}: \mathscr{P}_{\mathbb{K}, N} \rightarrow \mathscr{P}_{\mathbb{L}, N}
$$

such that $\chi(\mathfrak{p})=\breve{\psi}(\chi)\left(\varphi_{N}(\mathfrak{p})\right)$ for all $\chi \in \breve{G}_{\mathbb{K}}^{\text {ab }}$ and all $\mathfrak{p} \in \mathscr{P}_{\mathbb{K}, N}$. That suffices to prove (i), as we now first explain. We obtain a bijection

$$
\varphi: \mathscr{P}_{\mathbb{K}} \rightarrow \mathscr{P}_{\mathbb{L}}
$$

by setting $\left.\varphi\right|_{\mathscr{P}_{\mathbb{K}, N}}:=\varphi_{N}$, so that $\varphi$ satisfies $\chi(\mathfrak{p})=\breve{\psi}(\chi)(\varphi(\mathfrak{p}))$, for all $\chi \in \breve{G}_{\mathbb{K}}^{\text {ab }}$ and all $\mathfrak{p} \in \mathscr{P}_{\mathbb{K}}$. From this, the following sequence of equivalences follows:

$$
\mathfrak{p} \in U(\chi) \Longleftrightarrow \chi(\mathfrak{p}) \neq 0 \Longleftrightarrow \breve{\psi}(\chi)(\varphi(\mathfrak{p})) \neq 0 \Longleftrightarrow \varphi(\mathfrak{p}) \in U(\breve{\psi}(\chi)) .
$$

Because $\varphi$ is a bijection, we obtain $\varphi(U(\chi))=U(\breve{\psi}(\chi))$. Certainly $\varphi$ gives rise to a monoid isomorphism $I_{\mathbb{K}} \stackrel{\sim}{\rightarrow} I_{\mathbb{L}}$, and (1) follows from Lemma 2.2 combined with $\varphi(U(\chi))=$ $U(\breve{\psi}(\chi))$. Thus, for every $\mathfrak{p} \in \mathscr{P}_{\mathbb{K}}, \chi(\mathfrak{p})=\breve{\psi}(\chi)(\varphi(\mathfrak{p}))$ for all $\chi \in \breve{G}_{\mathbb{K}}^{\text {ab }}$ with $\mathfrak{p} \in U(\chi)$ implies that for any splits $s_{\mathbb{K}}$ and $s_{\mathbb{L}}$,

$$
\psi\left(\operatorname{rec}_{\mathbb{K}}\left(s_{\mathbb{K}}(\mathfrak{p})\right)\right) \equiv \operatorname{rec}_{\mathbb{L}}\left(s_{\mathbb{L}}(\varphi(\mathfrak{p}))\right) \bmod \operatorname{rec}_{\mathbb{L}}\left(\mathscr{O}_{\varphi(\mathfrak{p})}^{*}\right) .
$$

Hence we can modify our splits to obtain (2), and this proves (i).

To construct $\varphi_{N}$, the strategy is to proceed inductively on $N$. For $N=1$, the statement is empty. Assume that we have constructed $\varphi_{M}$ for all $M<N$. Let

$$
\varphi_{<N}: \mathscr{P}_{\mathbb{K},<N} \rightarrow \mathscr{P}_{\mathbb{L},<N}
$$

be the norm-preserving bijection obtained by combining the $\varphi_{M}$. Since

$$
U_{<N}(\breve{\psi}(\chi))=\varphi_{<N}\left(U_{<N}(\chi)\right), \chi(\mathfrak{p})=\breve{\psi}(\chi)\left(\varphi_{<N}(\mathfrak{p})\right) \text {, and } N(\mathfrak{p})=N\left(\varphi_{<N}(\mathfrak{p})\right),
$$


it follows that

$$
L_{<N}(\chi)=L_{<N}(\breve{\psi}(\chi)) .
$$

Since $L(\chi)=L(\breve{\psi}(\chi))$ and $L(\chi)=L_{<N}(\chi) L_{\geq N}(\chi)$ we also have

$$
L_{\geq N}(\chi)=L_{\geq N}(\breve{\psi}(\chi)) \text {. }
$$

We can apply this equality to prove the following lemma.

Lemma 6.2 For any character $\chi \in \breve{G}_{\mathbb{K}}^{\mathrm{ab}}$, define

$$
\mathscr{X}_{N}(\chi):=\sum_{\mathfrak{p} \in \mathscr{P}_{\mathbb{K}, N}} \chi(\mathfrak{p})=\sum_{\mathfrak{p} \in U_{N}(\chi)} \chi(\mathfrak{p}) .
$$

Then $\mathscr{X}_{N}(\chi)=\mathscr{X}_{N}(\breve{\psi}(\chi))$ for all $\chi \in \breve{G}_{\mathbb{K}}^{\mathrm{ab}}$. As a result, $\left|\mathscr{P}_{\mathbb{K}, N}\right|=\left|\mathscr{P}_{\mathbb{L}, N}\right|$.

Proof Let $\left\langle\mathscr{P}_{\mathbb{K}, \geq N}\right\rangle$ be the submonoid of $I_{\mathbb{K}}$ generated by $\mathscr{P}_{\mathbb{K}, \geq N}$. We write $L_{\geq N}(\chi)$ in additive form:

$$
L_{\geq N}(\chi)=\sum_{\mathfrak{m} \in\left\langle\mathscr{P}_{\mathbb{K}, \geq N}\right\rangle} \chi(\mathfrak{m}) N(\mathfrak{m})^{-s}=\sum_{M \geq N}\left(\sum_{\mathfrak{m} \in\left\langle\mathscr{P}_{\mathbb{K}, \geq N}\right\rangle \cap \cap_{\mathbb{K}, M}} \chi(\mathfrak{m})\right) M^{-s} .
$$

As $L_{\geq N}(\chi)=L_{\geq N}(\breve{\psi}(\chi))$, the coefficients of $N^{-s}$ in both sums are equal, hence

$$
\sum_{\mathfrak{m} \in\left\langle\mathscr{P}_{\mathbb{K}, \geq N}\right\rangle \cap I_{\mathbb{K}, N}} \chi(\mathfrak{m})=\sum_{\mathfrak{n} \in\left\langle\mathscr{P}_{\mathbb{L}, \geq N}\right\rangle \cap \mathbb{L}_{\mathbb{L}, N}} \check{\psi}(\chi)(\mathfrak{n}) .
$$

As $\left\langle\mathscr{P}_{\mathbb{K}, \geq N}\right\rangle \cap I_{\mathbb{K}, N}=\mathscr{P}_{\mathbb{K}, N}$ and $\left\langle\mathscr{P}_{\mathbb{L}, \geq N}\right\rangle \cap I_{\mathbb{L}, N}=\mathscr{P}_{\mathbb{L}, N}$, the desired equality follows. The final result is obtained by setting $\chi=1$.

Definition 6.3 Let $c_{N}:=\left|\mathscr{P}_{\mathbb{K}, N}\right|=\left|\mathscr{P}_{\mathbb{L}, N}\right|$. For any character $\chi \in \breve{G}_{\mathbb{K}}^{\mathrm{ab}}$, define

- $u_{N}(\chi)=\left|U_{N}(\chi)\right|$,

- $V_{N}(\chi)=\left\{\mathfrak{p} \in U_{N}(\chi) \mid \chi(\mathfrak{p})=1\right\}$, and

- $v_{N}(\chi)=\left|V_{N}(\chi)\right|$.

If $v_{N}(\chi)=u_{N}(\chi)-1$, there exists a unique prime of norm $N$ on which $\chi$ has a value of neither 0 nor 1 . We will denote this prime by $\mathfrak{p}_{\chi}$. Lastly, we define the following sets of characters:

$$
\begin{aligned}
& \Xi_{\mathbb{K}}^{1}:=\left\{\chi \in \breve{G}_{\mathbb{K}}^{\mathrm{ab}}: u_{N}(\chi)=v_{N}(\chi)=c_{N}\right\} \\
& \Xi_{\mathbb{K}}^{2}:=\left\{\chi \in \breve{G}_{\mathbb{K}}^{\mathrm{ab}}: u_{N}(\chi)=c_{N}, v_{N}(\chi)=c_{N}-1, \chi\left(\mathfrak{p}_{\chi}\right)=\zeta\right\},
\end{aligned}
$$

where $\zeta=\exp (2 \pi i / k)$ for some fixed integer $k \geq 3$.

Remark 6.4 Let $\chi \in \breve{G}_{\mathbb{K}}^{\text {ab }}$. As $|\chi(\mathfrak{p})|=1$ for all $\mathfrak{p} \in U_{N}(\chi)$, we have $\operatorname{Re}\left(\mathscr{X}_{N}(\chi)\right) \leq u_{N}(\chi)$. Equality holds precisely when $\chi(\mathfrak{p})=1$ for all $\mathfrak{p} \in U_{N}(\chi)$, i.e. $u_{N}(\chi)=v_{N}(\chi)$.

Lemma 6.5 $\breve{\psi}\left(\Xi_{\mathbb{K}}^{1}\right)=\Xi_{\mathbb{L}}^{1}$.

Proof From Lemma 6.2 we obtain $\chi \in \Xi_{\mathbb{K}}^{1} \Longleftrightarrow \mathscr{X}_{N}(\chi)=c_{N} \Longleftrightarrow \mathscr{X}_{N}(\breve{\psi}(\chi))=$ $c_{N} \Longleftrightarrow \check{\psi}(\chi) \in \Xi_{\mathbb{L}}^{1}$. 
Lemma 6.6 For $k \geq 3$, let $\mu_{k}$ denote the $k$ th roots of unity and let $\zeta=\exp (2 \pi i / k)$. Suppose we have $a_{1}, \ldots, a_{n} \in \mu_{k} \cup\{0\}$ such that $a_{1}+\cdots+a_{n}=n-1+\zeta$. Then there is a $j$ such that $a_{j}=\zeta$, and $a_{i}=1$ for all $i \neq j$.

Proof If $k=3$, then $\zeta$ is the only possible value with a positive imaginary part. Hence one of the $a_{j}$ equals $\zeta$, and then $a_{i}=1$ for the remaining $i \neq j$, since they have to sum to $n-1$. For $k>3$, let $R:=\max \operatorname{Re}\left(\mu_{k}-\{1\}\right)$, and let $f$ denote the number of $a_{i}$ that are not equal to 1 . Since $0 \leq R=\operatorname{Re}(\zeta)<1$, we find that

$$
\begin{aligned}
n-1+R & =n-1+\operatorname{Re}(\zeta)=\operatorname{Re}\left(a_{1}+\cdots+a_{n}\right) \\
& \leq n-f+f \operatorname{Re}(\zeta)=n+f(R-1),
\end{aligned}
$$

so $R-1 \leq f(R-1)$, hence $f \leq 1$.

Lemma $6.7 \breve{\psi}\left(\Xi_{\mathbb{K}}^{2}\right)=\Xi_{\mathbb{L}}^{2}$.

Proof For any character $\chi \in \Xi_{\mathbb{K}}^{2}$ we have $\chi^{k} \in \Xi_{\mathbb{K}}^{1}$. Thus, by Lemma $6.5, \breve{\psi}(\chi)^{k} \in \Xi_{\mathbb{L}}^{1}$. Hence for any $\mathfrak{q} \in \mathscr{P}_{\mathbb{L}, N}$ we have that $\breve{\psi}(\chi)(\mathfrak{q}) \in \mu_{k} \cup\{0\}$. As $\mathscr{X}_{N}(\breve{\psi}(\chi))=\mathscr{X}_{N}(\chi)=$ $c_{N}-1+\zeta$, by the previous lemma there exists a single prime $\mathfrak{q}_{\breve{\psi}(\chi)}$ such that $\breve{\psi}(\chi)\left(\mathfrak{q}_{\breve{\psi}(\chi)}\right)=$ $\zeta$, while $\breve{\psi}(\chi)(\mathfrak{q})=1$ for all $\mathfrak{q} \neq \mathfrak{q}_{\breve{\psi}(\chi)}$. Hence $\breve{\psi}\left(\Xi_{\mathbb{K}}^{2}\right) \subseteq \Xi_{\mathbb{L}}^{2}$. By symmetry, we have equality.

A character $\chi$ in $\Xi_{\mathbb{K}}^{2}$ has a special prime $\mathfrak{p}_{\chi}$ of norm $N$, and the corresponding character $\breve{\psi}(\chi) \in \Xi_{\mathbb{L}}^{2}$ has a special prime $\mathfrak{q}_{\breve{\psi}(\chi)}$ of norm $N$. We obtain a relation between primes

$$
\left\{\left(\mathfrak{p}_{\chi}, \mathfrak{q}_{\breve{\psi}(\chi)}\right): \chi \in \Xi_{\mathbb{K}}^{2}\right\} \subseteq \mathscr{P}_{\mathbb{K}, N} \times \mathscr{P}_{\mathbb{L}, N}
$$

which we will now show is the graph $\left\{\left(\mathfrak{p}_{\chi}, \varphi_{N}\left(\mathfrak{p}_{\chi}\right)\right\}\right.$ of a bijection $\varphi_{N}: \mathscr{P}_{\mathbb{K}, N} \rightarrow \mathscr{P}_{\mathbb{L}, N}$ with the required property. The first step is to show that every prime of $\mathscr{P}_{\mathbb{K}, N}$ is associated to at least one prime of $\mathscr{P}_{\mathbb{L}, N}$.

Lemma 6.8 For every $\mathfrak{p}^{\prime} \in \mathscr{P}_{\mathbb{K}, N}$ there exists a character $\chi \in \Xi_{\mathbb{K}}^{2}$ such that $\mathfrak{p}_{\chi}=\mathfrak{p}^{\prime}$.

Proof The Grunwald-Wang Theorem [2, Ch. X, Thm. 5] guarantees that there exists a character $\chi \in \breve{G}_{\mathbb{K}}^{\text {ab }}$ such that $\chi\left(\mathfrak{p}^{\prime}\right)=\zeta$ and $\chi(\mathfrak{p})=1$ for all primes $\mathfrak{p} \neq \mathfrak{p}^{\prime}$ of norm $N$, because there exists a character of $G_{\mathbb{K}_{\mathfrak{p}^{\prime}}}^{\text {ab }}$ whose fixed field is the unique unramified extension of degree $k$ of $\mathbb{K}_{\mathfrak{p}^{\prime}}$.

Lemma 6.9 The map $\varphi_{N}: \mathscr{P}_{\mathbb{K}, N} \rightarrow \mathscr{P}_{\mathbb{L}, N}: \mathfrak{p}_{\chi} \mapsto \mathfrak{q}_{\breve{\psi}(\chi)}$ is a well-defined bijection such that for every $\chi \in \breve{G}_{\mathbb{K}}^{\mathrm{ab}}$ and $\mathfrak{p} \in \mathscr{P}_{\mathbb{K}, N}$ we have $\chi(\mathfrak{p})=\breve{\psi}(\chi)\left(\varphi_{N}(\mathfrak{p})\right)$.

Proof Suppose we have $\chi, \chi^{\prime} \in \Xi_{\mathbb{K}}^{2}$ such that $\mathfrak{p}_{\chi}=\mathfrak{p}_{\chi^{\prime}}$ and $\mathfrak{q}_{\breve{\psi}(\chi)} \neq \mathfrak{q}_{\breve{\psi}\left(\chi^{\prime}\right)}$. We have

$$
\mathscr{X}_{N}\left(\chi \cdot \chi^{\prime}\right)=c_{N}-1+\zeta^{2},
$$

while

$$
\mathscr{X}_{N}\left(\breve{\psi}\left(\chi \cdot \chi^{\prime}\right)\right)=\mathscr{X}_{N}\left(\breve{\psi}(\chi) \cdot \breve{\psi}\left(\chi^{\prime}\right)\right)=c_{N}-2+2 \zeta \neq c_{N}-1+\zeta^{2},
$$

which contradicts Lemma 6.2. We conclude that $\mathfrak{q}_{\breve{\psi}(\chi)}=\mathfrak{q}_{\breve{\psi}\left(\chi^{\prime}\right)}$. Using $\breve{\psi}^{-1}$ instead of $\breve{\psi}$ provides a well-defined inverse $\varphi_{N}^{-1}$. 
Take $\chi^{\prime} \in \Xi_{\mathbb{K}}^{2}$ such that $\mathfrak{p}_{\chi^{\prime}}=\mathfrak{p}$. We have

$$
\mathscr{X}_{N}\left(\chi \cdot \chi^{\prime}\right)=\mathscr{X}_{N}(\chi)-\chi(\mathfrak{p})+\zeta \chi(\mathfrak{p})=\mathscr{X}_{N}(\chi)-(1-\zeta) \chi(\mathfrak{p}) .
$$

Similarly,

$$
\mathscr{X}_{N}\left(\breve{\psi}\left(\chi \cdot \chi^{\prime}\right)\right)=\mathscr{X}_{N}\left(\breve{\psi}(\chi) \cdot \breve{\psi}\left(\chi^{\prime}\right)\right)=\mathscr{X}_{N}(\breve{\psi}(\chi))-(1-\zeta) \breve{\psi}(\chi)\left(\varphi_{N}(\mathfrak{p})\right) .
$$

As we have both $\mathscr{X}_{N}(\chi)=\mathscr{X}_{N}(\breve{\psi}(\chi))$ and $\mathscr{X}_{N}\left(\chi \cdot \chi^{\prime}\right)=\mathscr{X}_{N}\left(\breve{\psi}\left(\chi \cdot \chi^{\prime}\right)\right)$, we conclude that $(1-\zeta) \chi(\mathfrak{p})=(1-\zeta) \breve{\psi}(\chi)\left(\varphi_{N}(\mathfrak{p})\right)$ and consequently $\chi(\mathfrak{p})=\breve{\psi}(\chi)\left(\varphi_{N}(\mathfrak{p})\right)$.

This completes the proof of Proposition 6.1.

\section{Conditional reconstruction of global fields}

Proposition 7.1 Assume the equivalent statements (i)-(iii) of Theorem 3.1. Then there exists an isomorphism $\Psi$ such that

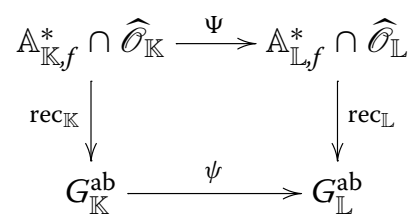

commutes.

Proof Define the homomorphism $\widehat{\mathscr{O}}_{\mathbb{K}}^{*} \times I_{\mathbb{K}} \rightarrow \mathbb{A}_{\mathbb{K}, f}^{*} \cap \widehat{\mathscr{O}}_{\mathbb{K}}$ by $(u, \mathfrak{m}) \mapsto u \cdot s_{\mathbb{K}}(\mathfrak{m})$. It has a complete inverse $\mathbb{A}_{\mathbb{K}, f}^{*} \cap \widehat{\mathscr{O}}_{\mathbb{K}} \rightarrow \widehat{\mathscr{O}}_{\mathbb{K}}^{*} \times I_{\mathbb{K}}$ given by $x \mapsto\left(x \cdot s_{\mathbb{K}}\left((x)_{\mathbb{K}}\right)^{-1},(x)_{\mathbb{K}}\right)$. We obtain an isomorphism $\mathbb{A}_{\mathbb{K}, f}^{*} \cap \widehat{\mathscr{O}}_{\mathbb{K}} \stackrel{\sim}{\rightarrow} \widehat{\mathscr{O}}_{\mathbb{K}}^{*} \times I_{\mathbb{K}}$ (and similarly for $\mathbb{L}$ ).

As seen in the proof of Proposition 4.1,

$$
\mathscr{O}_{\mathfrak{p}}^{*} \stackrel{\sim}{\longrightarrow} \operatorname{rec}_{\mathbb{K}}\left(\mathscr{O}_{\mathfrak{p}}^{*}\right) \stackrel{\psi}{\rightarrow} \operatorname{rec}_{\mathbb{L}}\left(\mathscr{O}_{\varphi(\mathfrak{p})}^{*}\right) \stackrel{\sim}{\rightarrow} \mathscr{O}_{\varphi(\mathfrak{p})}^{*}
$$

We obtain an isomorphism

$$
\Psi_{\mathfrak{p}}: \mathscr{O}_{\mathfrak{p}}^{*} \stackrel{\sim}{\longrightarrow} \mathscr{O}_{\varphi(\mathfrak{p})}^{*}
$$

that fits into the following commutative diagram:

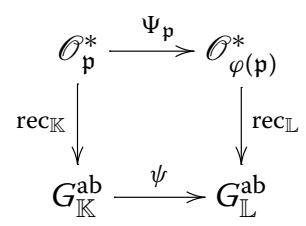

As we have $\psi\left(\operatorname{rec}_{\mathbb{K}}\left(s_{\mathbb{K}}(\mathfrak{m})\right)\right)=\operatorname{rec}_{\mathbb{L}}\left(s_{\mathbb{L}}(\varphi(\mathfrak{m}))\right)$ for all $\mathfrak{m} \in I_{\mathbb{K}}$ by assumption, the map $\left(\prod \Psi_{\mathfrak{p}}\right) \times \varphi$ fits in the commutative diagram

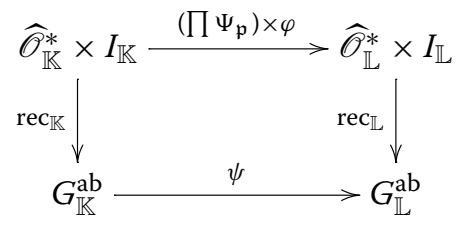

Using the aforementioned identifications $\mathbb{A}_{\mathbb{K}, f}^{*} \cap \widehat{\mathscr{O}}_{\mathbb{K}} \stackrel{\sim}{\rightarrow} \widehat{\mathscr{O}}_{\mathbb{K}}^{*} \times I_{\mathbb{K}}$ and $\mathbb{A}_{\mathbb{L}, f}^{*} \cap \widehat{\mathscr{O}}_{\mathbb{L}} \stackrel{\sim}{\rightarrow} \widehat{\mathscr{O}}_{\mathbb{L}}^{*} \times I_{\mathbb{L}}$ we obtain the desired isomorphism $\Psi$. 
Corollary 7.2 Diagram (5) can be extended to a commutative diagram

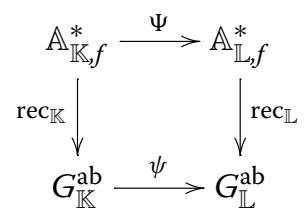

Proof The reciprocity map is already defined from $\mathbb{A}_{\mathbb{K}, f}^{*}$ to $G_{\mathbb{K}}^{\mathrm{ab}}$, so this follows from (5) by passing to the group of fractions $\mathbb{A}_{\mathbb{K}, f}^{*}$ of the monoid $\mathbb{A}_{\mathbb{K}, f}^{*} \cap \widehat{\mathscr{O}}_{\mathbb{K}}$.

We now turn to the reconstruction of isomorphism of fields from the equivalent conditions in the Main Theorem 3.1. For this, we first quote a result about conditions under which an isomorphism of multiplicative groups of fields can be extended to a field isomorphism. Let $\mathscr{O}_{[\mathfrak{p}]}$ denote the local ring (of $\mathbb{K}$ ) at the prime $\mathfrak{p}$.

Lemma 7.3 (Uchida/Hoshi) An isomorphism $F: \mathbb{K}^{*} \rightarrow \mathbb{L}^{*}$ of multiplicative groups of two global fields $\mathbb{K}$ and $\mathbb{L}$ is the restriction of an isomorphism of fields if and only if there exists a bijection $\varphi: \mathscr{P}_{\mathbb{K}} \rightarrow \mathscr{P}_{\mathbb{L}}$ such that for all $\mathfrak{p} \in \mathscr{P}_{\mathbb{K}}$, both the following hold:

(i) $F\left(1+\mathfrak{p} \mathscr{O}_{[\mathfrak{p}]}\right)=1+\varphi(\mathfrak{p}) \mathscr{O}_{[\varphi(\mathfrak{p})]}$ (as sets, or, equivalently, as subgroups),

(ii) $v_{\varphi(\mathfrak{p})} \circ F=v_{\mathfrak{p}}$.

Proof This follows immediately by results of Uchida for global function fields [22, Lemma $8-11]$, as explained in the introduction of [12]) and by Hoshi for number fields [12, Thm. D].

Theorem 7.4 Assume that $\Psi$ in (7) above satisfies $\Psi\left(\mathbb{K}^{*}\right)=\mathbb{L}^{*}$. Then the extension of that isomorphism to a map $\mathbb{K} \rightarrow \mathbb{L}$ by setting $0 \mapsto 0$ is an isomorphism of fields.

Proof Fix $\mathfrak{p} \in \mathscr{P}_{\mathbb{K}}$. Let $\Pi_{\mathfrak{p}}: \mathbb{A}_{\mathbb{K}, f}^{*} \rightarrow \mathbb{K}_{\mathfrak{p}}^{*}$ be the canonical projection, which we use to project elements of $\mathbb{K}^{*}$ (that are diagonally embedded in $\mathbb{A}_{\mathbb{K}, f}^{*}$ ) into the completions $\mathbb{K}_{\mathfrak{p}}^{*}$. By construction, there is an isomorphism $\Psi_{\mathfrak{p}}: \mathbb{K}_{\mathfrak{p}}^{*} \stackrel{\sim}{\rightarrow} \mathbb{L}_{\varphi(\mathfrak{p})}^{*}$ such that

$$
\Pi_{\varphi(\mathfrak{p})} \circ \Psi=\Psi_{\mathfrak{p}} \circ \Pi_{\mathfrak{p}}
$$

The one-units of the complete local ring are simply the $(N(\mathfrak{p})-1)$-th powers $[16, \mathrm{Ch}$. II, Prop. 5.7]:

$$
1+\pi_{\mathfrak{p}} \mathscr{O}_{\mathfrak{p}}=\left(\mathscr{O}_{\mathfrak{p}}^{*}\right)^{N(\mathfrak{p})-1}
$$

Since $\Psi_{\mathfrak{p}}$ is multiplicative, and respects units by Eq. (6) we find

$$
\Psi_{\mathfrak{p}}\left(1+\mathfrak{p} \mathscr{O}_{\mathfrak{p}}\right)=1+\varphi(\mathfrak{p}) \mathscr{O}_{\varphi(\mathfrak{p})}
$$

Setting $F:=\left.\Psi\right|_{\mathbb{K}^{*}}: \mathbb{K}^{*} \rightarrow \mathbb{L}^{*}$, we conclude that for the local rings, we have

$$
\begin{aligned}
F\left(1+\mathfrak{p} \mathscr{O}_{[\mathfrak{p}]}\right) & =\Psi\left(\mathbb{K}^{*} \cap \Pi_{\mathfrak{p}}^{-1}\left(1+\mathfrak{p} \mathscr{O}_{\mathfrak{p}}\right)\right) \\
& =\Psi\left(\mathbb{K}^{*}\right) \cap \underbrace{\Psi \circ \Pi_{\mathfrak{p}}^{-1}}_{=\Pi_{\varphi(\mathfrak{p})}^{-1} \Psi_{\mathfrak{p}}}\left(1+\mathfrak{p} \mathscr{O}_{\mathfrak{p}}\right) \\
& =\mathbb{L}^{*} \cap \Pi_{\varphi(\mathfrak{p})}^{-1}\left(1+\varphi(\mathfrak{p}) \mathscr{O}_{\varphi(\mathfrak{p})}\right)
\end{aligned}
$$




$$
=1+\varphi(\mathfrak{p}) \mathscr{O}_{[\varphi(\mathfrak{p})]} \cdot
$$

This proves condition (i) in Lemma 7.3.

For condition (ii), observe that from the definition of $\Psi$ it follows that $\Psi\left(s_{\mathbb{K}}(\mathfrak{p})\right)=$ $s_{\mathbb{L}}(\varphi(\mathfrak{p}))$. Since $\Pi_{\mathfrak{p}}\left(s_{\mathbb{K}}(\mathfrak{p})\right)=\pi_{\mathfrak{p}}$ by definition of a split (for a chosen uniformizer $\pi_{\mathfrak{p}}$ ), we find that

$$
\Psi_{\mathfrak{p}}\left(\pi_{\mathfrak{p}}\right)=\Pi_{\phi(\mathfrak{p})}\left(s_{\mathbb{L}}(\varphi(\mathfrak{p}))\right)=: \pi_{\varphi(\mathfrak{p})}
$$

is a uniformizer at $\varphi(\mathfrak{p})$. Thus, the multiplicative map $F$ respects elements of valuation 0 (see again Eq. (6)) and 1, and hence (ii) holds.

\section{Reconstruction of global function fields}

In function fields, we can immediately apply the results of the previous section:

Proposition 8.1 If one of the equivalent conditions (i)-(iii) of Theorem 3.1 holds for two global function fields $\mathbb{K}$ and $\mathbb{L}$, then they are isomorphic as fields.

Proof From the commutativity of diagram (7) we find that $\Psi\left(\operatorname{ker}\left(\operatorname{rec}_{\mathbb{K}}\right)\right)=\operatorname{ker}\left(\operatorname{rec}_{\mathbb{L}}\right)$. For a global function field $\mathbb{K}$ we have $\operatorname{ker}\left(\operatorname{rec}_{\mathbb{K}}\right)=\mathbb{K}^{*}$ and therefore the result follows from Theorem 7.4.

Remark 8.2 The equivalence of (iii) in Theorem 3.1 and field isomorphism in global function fields was also shown in [3] using dynamical systems, but referring to the unpublished [4] for a proof of the result in Sect. 6 of this paper.

\section{The number field case: an auxiliary result}

In this section we prove the existence of certain Galois extensions of number fields with prescribed Galois groups; a result that we will use in the next section to prove the reconstruction of number fields from the consideration of specific induced representations.

Proposition 9.1 Let $\mathbb{K}$ be a number field of degree $n$ contained in a finite Galois extension $\mathbb{N}$ of $\mathbb{Q}$, and let $C$ be a finite cyclic group. Denote $G=\operatorname{Gal}(\mathbb{N} / \mathbb{Q})$ and $H=G a l(\mathbb{N} / \mathbb{K})$ and let $C^{n} \rtimes G$ be the semidirect product of $C^{n}$ and $G$, where the action of $G$ on $C^{n}$ is by permuting coordinates the same way $G$ permutes the cosets $G / H$. By $C^{n} \rtimes H$ we denote the subgroup of $C^{n} \rtimes G$ generated by $C^{n}$ and $H$. There exists a Galois extension $\mathbb{M}$ of $\mathbb{Q}$ containing $\mathbb{N}$ such that

$$
\operatorname{Gal}(\mathbb{M} / \mathbb{Q})=C^{n} \rtimes G, \operatorname{Gal}(\mathbb{M} / \mathbb{K})=C^{n} \rtimes H, \text { and } \operatorname{Gal}(\mathbb{M} / \mathbb{N})=C^{n} .
$$

Remark 9.2 The semidirect product $C^{n} \rtimes G$ is also known as the wreath product of $C$ and the group $G$ considered as a permutation group on $G / H$. For any extension $\mathbb{L}$ of $\mathbb{K}$ with Galois group $C$, the Galois group of the Galois closure of $\mathbb{L}$ over $\mathbb{Q}$ is a subgroup of this wreath product. The proposition asserts that the wreath product itself (i.e., the maximal subgroup, which can be viewed as the 'generic' case), actually occurs for some $\mathbb{L}$.

We give a self-contained proof, but the result also follows from [14, Thm. IV.2.2]; or, for $C$ of order 3 one can use the existence of a generic polynomial for $C$ and apply [7, Prop. 13.8.2].

Proof of Proposition 9.1 Let $p \neq 2$ be a prime that is totally split in $\mathbb{N}$ and denote by $\mathfrak{p}_{1}, \ldots, \mathfrak{p}_{n}$ the primes in $\mathbb{K}$ lying above $p$. There exists a Galois extension $\widetilde{\mathbb{K}} / \mathbb{K}$ with Galois 
group $C$ in which the prime $\mathfrak{p}_{1}$ is inert, while $\mathfrak{p}_{2}, \ldots, \mathfrak{p}_{n}$ are totally split (this follows, e.g., from the Grunwald-Wang theorem).

Let $X$ be the set of field homomorphisms from $\mathbb{K}$ to $\mathbb{N}$. Since $G$ acts on $\mathbb{N}$, we get an action of $G$ on $X$ by composition. This action is transitive and the stabilizer of the inclusion map $\iota \in X$ is $H$, so $X$ is isomorphic to $G / H$ as a $G$-set. For each $\sigma \in X$ we now consider $\widetilde{\mathbb{N}}_{\sigma}:=\widetilde{\mathbb{K}} \otimes_{\mathbb{K}, \sigma} \mathbb{N}$, where $\mathbb{N}$ is viewed as a $\mathbb{K}$-algebra through $\sigma: \mathbb{K} \rightarrow \mathbb{N}$. The $C$-action on $\widetilde{\mathbb{K}}$ induces a $C$-action on $\widetilde{\mathbb{N}}_{\sigma}$ by $\mathbb{N}$-algebra automorphisms. Setting $P_{\sigma}$ to be the set of primes of $\mathbb{N}$ that contain $\sigma\left(\mathfrak{p}_{1}\right)$, we see that $\widetilde{\mathbb{N}}_{\sigma}$ is a Galois extension of $\mathbb{N}$ with Galois group $C$ for which the primes in $P_{\sigma}$ are inert and all other primes of $\mathbb{N}$ over $p$ are totally split.

The $G$-action on the set of primes of $\mathbb{N}$ over $p$ is free and transitive, and $P_{l}$ consists of a single $H$-orbit: the primes over $\mathfrak{p}_{1}$. Since $P_{g \sigma}=g P_{\sigma}$ it follows that as $\sigma$ ranges over $X$ the sets $P_{\sigma}$ form a disjoint family. One deduces that the fields $\widetilde{\mathbb{N}}_{\sigma}$ form a linearly disjoint family of $C$-extensions of $\mathbb{N}$ and that the tensor product

$$
\mathbb{M}=\bigotimes_{\sigma \in X} \tilde{\mathbb{N}}_{\sigma}
$$

over $\mathbb{N}$ of all $\widetilde{\mathbb{N}}_{\sigma}$ with $\sigma \in X$ is a field which is Galois over $\mathbb{N}$ with Galois group $C^{n}=$ $\prod_{\sigma \in X} C$.

For $g \in G$ and $\sigma \in X$ there is a natural field isomorphism $\widetilde{g}_{\sigma}: \widetilde{\mathbb{N}}_{\sigma} \rightarrow \widetilde{\mathbb{N}}_{g \sigma}$ given by $x \otimes y \mapsto x \otimes g y$ that extends the map $\mathbb{N} \rightarrow \mathbb{N}$ given by $y \mapsto g y$. Combining these maps for all $\sigma \in X$ we obtain an automorphism of the tensor product $\mathbb{M}$ that permutes the factors of the tensor product by the $g$-action on $X$. Thus, we have extended the $G$-action on $\mathbb{N}$ to a $G$-action on $\mathbb{M}$. Since each $\widetilde{g}_{\sigma}$ is $C$-equivariant, the subgroup of Aut $(\mathbb{M})$ generated by $G$ and $C^{n}$ is the semidirect product $C^{n} \rtimes G$. As the cardinality of this group is the field degree of $\mathbb{M}$ over $\mathbb{Q}$ we see that $\mathbb{M}$ is a Galois extension of $\mathbb{Q}$ with Galois group $C^{n} \rtimes G$, and that $\mathbb{K}$ is the invariant field of $C^{n} \rtimes H$.

\section{Characterization of number fields}

Using the previous section we prove a stronger version of Proposition 8.1 for number fields.

Theorem 10.1 Let $\mathbb{K}$ be a number field and let $k \geq 3$. Then there exists a character $\chi \in \breve{G}_{\mathbb{K}}^{\mathrm{ab}}$ of order $k$ such that every number field $\mathbb{L}$ for which there is a character $\chi^{\prime} \in \breve{G}_{\mathbb{L}}^{\mathrm{ab}}$ with $L_{\mathbb{L}}\left(\chi^{\prime}\right)=L_{\mathbb{K}}(\chi)$ is isomorphic to $\mathbb{K}$.

We will use the following basic facts about Artin $L$-series of representations of the absolute Galois group $G_{\mathbb{K}}:=\operatorname{Gal}(\overline{\mathbb{Q}} / \mathbb{K})$ for a number field $\mathbb{K}$ within a fixed algebraic closure $\overline{\mathbb{Q}}$ of $\mathbb{Q}$.

Lemma 10.2 (a) For any two representations $\rho$ and $\rho^{\prime}$ of $G_{\mathbb{Q}}, L_{\mathbb{Q}}(\rho)=L_{\mathbb{Q}}\left(\rho^{\prime}\right)$ is equivalent to $\rho \stackrel{\sim}{\rightarrow} \rho^{\prime}$.

(b) For $\chi \in \check{G}_{\mathbb{K}}^{\mathrm{ab}}$, we have $L_{\mathbb{K}}(\chi)=L_{\mathbb{Q}}\left(\operatorname{Ind}_{G_{\mathbb{K}}}^{G_{\mathbb{Q}}}(\chi)\right)$.

(c) For any two number fields $\mathbb{K}$ and $\mathbb{L}$ within $\overline{\mathbb{Q}}$ and characters $\chi \in \check{G}_{\mathbb{K}}^{\text {ab }}$ and $\chi^{\prime} \in \check{G}_{\mathbb{L}}^{\text {ab }}$ with $L_{\mathbb{K}}(\chi)=L_{\mathbb{L}}\left(\chi^{\prime}\right)$ we have an isomorphism of representations of $G_{\mathbb{Q}}$

$$
\operatorname{Ind}_{G_{\mathbb{K}}}^{G \mathbb{Q}}(\chi) \stackrel{\sim}{\rightarrow} \operatorname{Ind}_{G_{\mathbb{L}}}^{G \mathbb{Q}}\left(\chi^{\prime}\right)
$$

and the fixed fields $\mathbb{K}_{\chi}$ of $\chi$ and $\mathbb{L}_{\chi^{\prime}}$ of $\chi^{\prime}$ have the same normal closure over $\mathbb{Q}$. 
Proof Fact (a) follows from Chebotarev's density theorem, comparing Euler factors. The basic fact (b) is due to Artin, see [16, VII.10.4.(iv)]. The isomorphism in (c) follows from (a) and (b). The last statement follows from the fact that the normal closure of $\mathbb{K}_{\chi}$ over $\mathbb{Q}$ is the fixed field of the kernel of the representation $\operatorname{Ind}_{G_{\mathbb{K}}}^{G_{\mathbb{Q}}}(\chi)$.

By a monomial structure of a representation $\rho$ of a group $G$ we mean a $G$-set $\mathscr{L}$ consisting of 1-dimensional subspaces of $\rho$ that is $G$-stable (i.e. $g L \in \mathscr{L}$ for all $g \in G$ and $L \in \mathscr{L}$ ), and such that as a vector space we have $\rho=\bigoplus_{L \in \mathscr{L}} L$. By choosing a single nonzero vector of $L$ for each $L \in \mathscr{L}$ one obtains a basis of $\rho$ such that for every $g \in G$ the action of $g$ on $\rho$ is given by a matrix with exactly one non-zero element in each row and in each column. If $H$ is a subgroup of $G$ and $\chi$ a linear character of $H$, then the induced representation $\rho=\operatorname{Ind}_{H}^{G}(\chi)$ naturally produces a monomial structure $\mathscr{L}$ that is isomorphic to $G / H$ as a $G$-set.

Proof of Theorem 10.1 If $L_{\mathbb{L}}\left(\chi^{\prime}\right)=L_{\mathbb{K}}(\chi)$ for two characters $\chi \in \check{G}_{\mathbb{K}}^{\text {ab }}, \chi^{\prime} \in \breve{G}_{\mathbb{L}}^{\text {ab }}$, the lemma implies that $\operatorname{Ind}_{G_{\mathbb{K}}}^{G_{\mathbb{Q}}}(\chi)$ has two monomial structures, one arising from $\chi$ and one from $\chi^{\prime}$. We see that $\mathbb{K}$ and $\mathbb{L}$ are isomorphic as number fields if and only if these two monomial structures are isomorphic as $G_{\mathbb{Q}^{-}}$sets (note that they are transitive $G_{\mathbb{Q}^{-}}$-sets). In order to prove Theorem 10.1 it therefore suffices to choose $\chi$ in such a way that the representation $\operatorname{Ind}_{G_{\mathbb{K}}}^{G_{\mathbb{Q}}}(\chi)$ only has a single monomial structure.

In order to find such a character $\chi$ we apply Proposition 9.1 to any finite Galois $\mathbb{N} / \mathbb{Q}$ containing $\mathbb{K}$, and where $C=\langle\zeta\rangle$ is the subgroup of $\mathbb{C}^{\times}$generated by $\zeta=\exp (2 \pi i / k)$. We let $n, G, H$, and $C^{n} \rtimes G$ be as in the proposition, and get an extension $\mathbb{M}$ of $\mathbb{K}$ within $\overline{\mathbb{Q}}$ with Galois group $\operatorname{Gal}(\mathbb{M} / \mathbb{Q})=C^{n} \rtimes G$. We order the coordinates of $C^{n}$ in such a way that the action of $H$ on $C^{n}$ fixes the first coordinate, so the map

$$
\operatorname{Gal}(\mathbb{M} / \mathbb{K})=C^{n} \rtimes H \rightarrow \mathbb{C}^{\times}:\left(a_{1}, \ldots, a_{n}, h\right) \mapsto a_{1}
$$

is a group homomorphism, and extends to a character $\chi \in \breve{G}_{\mathbb{K}}^{\mathrm{ab}}$. The induced representation $\rho=\operatorname{Ind}_{G_{\mathbb{K}}}^{G \mathbb{Q}}(\chi)$ factors over $\operatorname{Gal}(\mathbb{M} / \mathbb{Q})=C^{n} \rtimes G$ and it comes with a monomial structure $\mathscr{L}=\left\{L_{1}, \ldots, L_{n}\right\}$ such that each element $\left(a_{1}, \ldots, a_{n}\right) \in C^{n}$ acts on $L_{i}$ as scalar multiplication by $a_{i}$. It follows that $\mathscr{L}$ is exactly the set of 1 -dimensional $C^{n}$-submodules of $\rho$, the so-called character eigenspaces for the action of $C^{n}$ on $\rho$.

To finish the proof we will show that $\mathscr{L}$ is the unique monomial structure on the representation $\rho$. Suppose that $\mathscr{M}$ is another monomial structure on $\rho$. The trace of the element $c=(\zeta, 1, \ldots, 1) \in C^{n}$ on $\rho$ is equal to $n-1+\zeta$. On the other hand, $c$ permutes the elements of $\mathscr{M}$, so the trace of $c$ on $\rho$ is also equal to the sum of $k$-th roots of unity $\zeta_{M} \in \mu_{k}$ where $M$ ranges over those lines $M \in \mathscr{M}$ with $c M=M$, and $\zeta_{M}$ is the scalar by which $c$ then acts on $M$. Since $k \geq 3$ and $\mathscr{L}$ and $\mathscr{M}$ have the same number of elements, Lemma 6.6 implies that $c M=M$ for all $M \in \mathscr{M}$. It follows that $c$ acts trivially on the set $\mathscr{M}$. Since the G-conjugates of $c$ generate $C^{n}$ we deduce that $C^{n}$ acts trivially on the set $\mathscr{M}$. Thus, $\mathscr{M}$ consists of 1 -dimensional $C^{n}$-submodules of $\rho$. This implies that $\mathscr{M} \subset \mathscr{L}$, so $\mathscr{M}=\mathscr{L}$ for cardinality reasons.

Remark 10.3 Not every representation has a unique monomial structure: consider the isometry group of a square, the dihedral group $D_{4}$ of order 8, with its standard 2dimensional representation. It has two distinct monomial structures (consisting of the axes and the diagonals) and these are not isomorphic as $D_{4}$-sets. 
By realizing $D_{4}$ as a Galois group over $\mathbb{Q}$ this gives rise to quadratic fields $\mathbb{K}$ and and $\mathbb{L}$ with quadratic characters $\chi \in \breve{G}_{\mathbb{K}}^{\mathrm{ab}}[2]$ and $\chi^{\prime} \in \breve{G}_{\mathbb{L}}^{\mathrm{ab}}[2]$ that satisfy $L_{\mathbb{K}}(\chi)=L_{\mathbb{L}}\left(\chi^{\prime}\right)$ while $\mathbb{K}$ is not isomorphic to $\mathbb{L}$. This shows that the method of proof of the theorem fails without the assumption that $k \geq 3$. Concretely, $\operatorname{Gal}(\mathbb{Q}(\sqrt[4]{2}, i) / \mathbb{Q}) \stackrel{\sim}{\rightarrow} D_{4}$, and we find $L_{\mathbb{K}}(\chi)=L_{\mathbb{L}}\left(\chi^{\prime}\right)$ for $\mathbb{K}=\mathbb{Q}(\sqrt{2}), \mathbb{L}=\mathbb{Q}(i \sqrt{2})$ and $\chi$ and $\chi^{\prime}$ uniquely determined by $\mathbb{K}_{\chi}=\mathbb{Q}(\sqrt[4]{2})$ and $\mathbb{L}_{\chi^{\prime}}=\mathbb{Q}(i \sqrt{2},(1+i) \sqrt[4]{2})$.

In [18, Thm. 3.2.2] it is shown that $\mathbb{K}=\mathbb{Q}(\sqrt[8]{5})$ provides a counterexample to the statement of the theorem for $k=2$. On the other hand, in [18, Thm. 2.2.2], a similar method as in our proof is used to show that every number field is characterized uniquely by the $L$-series of $t$ wo suitable quadratic characters.

\section{Comparison of different methods of proof}

There is an interesting "incompatibility of proof techniques" between the case of global function fields and that of number fields. Namely, the approach of the proof of Proposition 8.1 for function fields does not transfer in an obvious way to number fields. Indeed, for a number field $\mathbb{K}, \operatorname{ker}\left(\operatorname{rec}_{\mathbb{K}}\right)=\mathbb{K}^{*} \cdot \overline{\mathscr{O}_{\mathbb{K},+}^{*}}$, where $\overline{\mathscr{O}_{\mathbb{K},+}^{*}}$ is the closure of the totally positive units (i.e., units of $\mathscr{O}_{\mathbb{K}}$ that are positive in every real embedding of $\mathbb{K}$ ) in the finite ideles; this follows from the description of the connected component of the idele class group by Artin [2, Ch. IX]. Hence the method of proof of 8.1 transferred literally to number fields yields the weaker conclusion that

$$
\Psi\left(\mathbb{K}^{*} \cdot \overline{\mathscr{O}_{\mathbb{K},+}^{*}}\right)=\mathbb{L}^{*} \cdot \overline{\mathscr{O}_{\mathbb{L},+}^{*}}
$$

It is unclear to us whether one can deduce that $\Psi\left(\mathbb{K}^{*}\right)=\mathbb{L}^{*}$ from the conditions in Theorem 3.1. The issue is similar to the one raised in [12, 3.3.2].

On the other hand, it is not possible to copy the proof of Theorem 10.1 for function fields, as this would force fixing a rational subfield $\mathbb{F}_{q}(t)$ inside both $\mathbb{K}$ and $\mathbb{L}$ (that plays the role of $\mathbb{Q}$ in the number field proof), for which there are infinitely many, non-canonical, choices. However, Theorem 10.1 does hold in the relative setting of separable geometric extensions of a fixed rational function field of characteristic not equal to 2, compare [19]. It is unclear to us whether the analogue of Theorem 10.1 holds for a global function field without fixing a rational subfield. It does seem that $L$-series of global function fields, as polynomials in $q^{-s}$, contain less arithmetical information than their number field cousins (compare [11]).

\section{Author details}

${ }^{1}$ Mathematisch Instituut, Universiteit Utrecht, Postbus 80.010, 3508 TA Utrecht, The Netherlands, ${ }^{2}$ Mathematisch Instituut, Universiteit Leiden, Postbus 9512, 2300 RA Leiden, The Netherlands, ${ }^{3}$ School of Mathematical Sciences, Queen Mary University of London, Mile End Road, London E1 4NS, UK, ${ }^{4}$ Mathematics Department, Caltech, Mail Code 253-37, 1200 E. California Blvd., Pasadena, CA 91125, USA, ${ }^{5}$ Perimeter Institute for Theoretical Physics, 31 Caroline Street North, Waterloo, ON N2L 2Y5, Canada, ${ }^{6}$ Department of Mathematics, University of Toronto, Bahen Centre, 40 St. George St., Toronto, ON M5S 2E4, Canada.

1. Angelakis, A., Stevenhagen, P.: Imaginary quadratic fields with isomorphic abelian Galois groups. In: Howe, E.W., Kedlaya, K.S. (eds.) ANTS X: Proceedings of the Tenth Algorithmic Number Theory Symposium. The Open Book Series, vol. 1. MSP, Berkeley, pp. 21-39 (2013). https://doi.org/10.2140/obs.2013.1.21

2. Artin, E., Tate, J.: Class Field Theory. AMS Chelsea Publishing, Providence (2008) 
3. Cornelissen, G.: Curves, dynamical systems, and weighted point counting. Proc. Natl Acad. Sci. U.S.A. 110, 9669-9673 (2013)

4. Cornelissen, G., Marcolli, M.: Quantum statistical mechanics, L-series and anabelian geometry. Preprint (2010). https:// arxiv.org/abs/1009.0736

5. Cornelissen, G., Marcolli, M.: Quantum statistical mechanics, L-series and anabelian geometry I: partition functions. In: Trends in Contemporary Mathematics. INdAM Series, vol. 8, p. 10. Springer, Heidelberg (2014)

6. Cornelissen, G., Li, X., Marcolli, M., Smit, H.: Reconstructing global fields from dynamics in the abelianized Galois group. Preprint (2017). https://arxiv.org/abs/1706.04517

7. Fried, M., Jarden, M.: Field arithmetic. In: Ergebn. Math. Grenzgeb. (3), vol. 11, 3rd edn. Springer, Berlin (2008)

8. Funakura, T.: Characters and Artin L-functions. Comment. Math. Univ. St. Paul. 27(2), 185-197 (1978/1979). http://id. nii.ac.jp/1062/00010285

9. Funakura, T.: Characters and Artin L-functions II. Comment. Math. Univ. St. Paul. 28(1), $81-85$ (1980). http://id.nii.ac.jp/ 1062/00010271

10. Gaßmann, F.: Bemerkungen zur Vorstehenden Arbeit von Hurwitz: Über Beziehungen zwischen den Primidealen eines algebraischen Körpers und den Substitutionen seiner Gruppen. Math. Z. 25, 661 (665)-675 (1926)

11. Gross, B.H.: Trivial L-functions for the rational function field. J. Number Theory 133, 970-976 (2013)

12. Hoshi, Y:: On the field-theoreticity of homomorphisms between the multiplicative groups of number fields. Publ. RIMS Kyoto Univ. 50, 269-285 (2014)

13. Kubota, T.: Galois group of the maximal abelian extension over an algebraic number field. Nagoya Math. J. 12, 177-189 (1957)

14. Malle, G., Matzat, B.H.: Inverse Galois Theory. Springer Monographs in Mathematics. Springer, Berlin (1999)

15. Neukirch, J.: Kennzeichnung der $p$-adischen und der endlichen algebraischen Zahlkörper. Invent. Math. 6, 296-314 (1969)

16. Neukirch, J.: Algebraic Number Theory. Grundlehren der Mathematischen Wissenschaften, vol. 322. Springer, Berlin (1999)

17. Onabe, M.: On the isomorphisms of the Galois groups of the maximal Abelian extensions of imaginary quadratic fields. Nat. Sci. Rep. Ochanomizu Univ. 27(2), 155-161 (1976). https://ci.nii.ac.jp/naid/110006559055/en/

18. Pintonello, M.: Characterizing number fields with quadratic L-functions. ALGANT Master Thesis in Mathematics, Università degli studi di Padova \& Universiteit Leiden, 25 June 2018. https://www.universiteitleiden.n//binaries/ content/assets/science/mi/scripties/master/algant/2017-2018/pintonello-master-thesis.pdf

19. Solomatin, P.: On Artin L-functions and Gassmann equivalence for global function fields. Preprint (2016). https://arxiv. org/abs/1610.05600

20. Tate, J.: Endomorphisms of abelian varieties over finite fields. Invent. Math. 2, 134-144 (1966)

21. Uchida, K.: Isomorphisms of Galois groups. J. Math. Soc. Jpn. 28(4), 617-620 (1976)

22. Uchida, K.: Isomorphisms of Galois groups of algebraic function fields. Ann. Math. (2) 106(3), 589-598 (1977) 